\title{
Pain relief with sphenopalatine ganglion block of chronic headache and orofacial postsurgical pain: Case Report
}

Maria Soares, Marta Pacheco, Ana Margarida Regalado, Carina Raposo, Asdrúbal Pinto, Isabel Santos

\author{
Serviço de Anestesiologia, Unidade Funcional de Dor Crónica \\ Centro Hospitalar do Porto
}

\section{Background}

- Chronic headache and orofacial pain is a common and disabling problem in patients with head injury or trauma and may be accompanied by autonomic, motor or sensory signs.

- Conventional treatments may fail or cause intolerable side effects.

- The Sphenopalatine Ganglion (SPG) block is a reasonable target for the treatment of these disorders.

- We report a case of chronic headache and orofacial post surgical pain and the role of SPG block on pain management.

\section{Case Report}

- A 37-year-old-female

- Background history:

Dentofacial dysmorphia, maxillary hyperplasia, mandibular hypoplasia and a LeFort I osteotomy in 2012.

\section{3}

- Chronic headache and orofacial pain since $2012 \rightarrow$ Chronic Pain Unit

$>$ Pain characterization:

- Hemicraneal - onset in the ipsilateral temporomandibular area;

- Continuous with exacerbations $\rightarrow$ NRS: 6-10

- Burning and electric shock, allodynia, paresthesia

- DN4 = 5

- Ipsilateral oculofacial autonomic phenomena

\section{$2013-2017$}

Several treatments were performed: gabapentinoids, weak opioids, antidepressants, acupuncture, topical treatment with Capsaicin $8 \%$ and Lidocaine $5 \% \rightarrow$ Minimally important pain relief.

\section{7 - SPG block:}

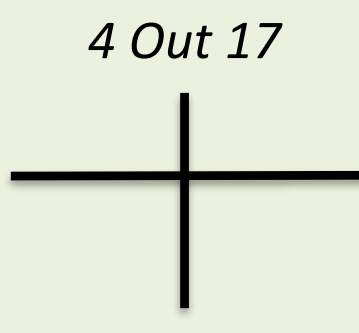

Ropi $0,75 \%$ NRS $8 \rightarrow 2$ (3 days)

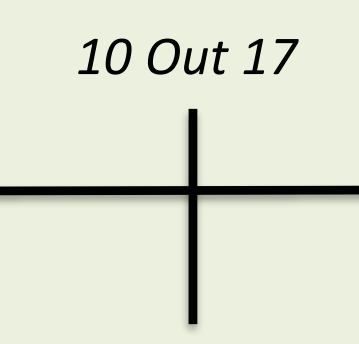

Ropi $0,75 \%$

NRS $8 \rightarrow 2$ (4 days)

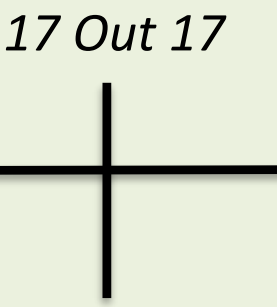

Ropi $0,75 \%$ NRS $8 \rightarrow 2$ (5 days)

- Total SPG block: 9

- Neurology: Sluder's Neuralgia $\rightarrow$ Tx: Topiramate, 50 mg twice daily

- Currantly: Acupuncture and SPG block every 4 to 6 weeks with sustained pain relief.

\section{Discussion}

- Nerves involved in headache and orofacial pain, pass through the SPG.

- SPG has an important autonomic component.

- SPG block interfere with parasympathetic outflow $\rightarrow$ principal mechanism for pain relief.

\section{Learning points}

- The SPG may be an important contributor to the pathogenesis of several headache and orofacial pain disorders.

- The SPG block is a safe and well tolerated technique, presenting good results in pain control. 